\title{
Extração de compostos fenólicos e atividade antioxidante em subprodutos da industrialização da polpa de araçá Ya-Cy (Psidium cattleianum Sabine)
}

\section{RESUMO}

Juliano Zanela

julianozanela@gmail.com

Universidade Tecnológica Federal do Paraná, Câmpus Dois Vizinhos, Dois Vizinhos, Paraná, Brasil.

\section{Maira Casagrande} mairacasagrande1@gmail.com Universidade Tecnológica Federal do Paraná, Câmpus Dois Vizinhos, Dois Vizinhos, Paraná, Brasil.

Américo Wagner Júnior americowagner@utfpr.edu.br Paraná Câmpus Dois Vizinhos, Dois Vizinhos, Paraná, Brasil.

\section{Luciano Lucchetta} lucianolucchetta@gmail.com Universidade Tecnológica Federal do Paraná, Câmpus Francisco Beltrão, Francisco Beltrão, Paraná, Brasil.

\section{Josiane Freitas Chim}

josianechim@gmail.com Universidade Federal de Pelotas, Pelotas, Rio Grande do Sul, Brasil.
O araçá (Psidium Cattleianum Sabine) é uma fruta nativa do Brasil ainda pouco explorada, porém estudos tem demonstrado que o araçá é uma potencial fonte de compostos bioativos, o que tem estimulado seu consumo, tanto na forma in natura como processado. O objetivo desse trabalho foi a extração dos compostos bioativos presentes nos subprodutos da extração da polpa (cascas e sementes). Frutos de araçá Ya-Cy foram coletados, sanitizados e despolpados, os subprodutos da extração das polpas foram liofilizados, moídos e posteriormente extraídos utilizando-se um planejamento fatorial $2^{2}$ com triplicata no ponto central. As variáveis independentes avaliadas foram: temperatura e tempo de extração: 40, 60 e $80^{\circ} \mathrm{C}$ e 30, 60 e 90 minutos, respectivamente. O solvente utilizado tratou-se de uma mistura ternária de água:etanol:acetona 1:1:1 (v/v). O conteúdo de compostos fenólicos e de ABTS variou de 11,74 a 13,35 mg de GAE.g ${ }^{-1}$ e 155,49 a 184,43 $\mu \mathrm{mol}$ TEAC. $\mathrm{g}^{-1}$ respectivamente, os valores de DPPH variaram de 90,16 a 108,82 $\mu \mathrm{mol}$ trolox. $g^{-1}$, já o FRAP não apresentou bom ajuste ao modelo, apresentando valor médio de 375,69 micromol Fe(II).g $\mathrm{g}^{-1}$. O subproduto da produção de polpa do araçá Ya-Cy se mostrou uma potencial fonte de compostos bioativos com atividade antioxidante.

PALAVRAS-CHAVE: Planejamento fatorial; compostos bioativos; araçá Ya-Cy. 


\section{INTRODUÇÃO}

O Brasil possui uma grande variedade de fruteiras nativas com potencial quase inexplorado. Dentre essas frutas, pode-se citar o araçá que aos poucos vem ganhando espaço, principalmente por meio de agroindústrias e cooperativas.

O araçá é uma frutífera nativa do Brasil, pertencente à família myrtaceae, amplamente distribuída desde o Estado de Minas Gerais até o Uruguai (MATTOS, 1989; RASEIRA; RASEIRA, 1996). No Brasil não há pomares comerciais de araçá visando o uso industrial, sendo considerado ainda um fruto silvestre, consumido principalmente in natura (HAMINIUK et al., 2006). De acordo com Antunes (2013), esse cenário vem mudando, pois, frutas como araçá, além de apresentar potencial para cultivo, vêm chamando a atenção devido as suas propriedades funcionais, por ser um alimento rico em compostos bioativos. Com isso, já se tem no mercado araçá do cultivar $\mathrm{Ya}-\mathrm{Cy}$, resultado do programa de melhoramento genético da Embrapa - Clima Temperado (Pelotas - RS).

A agro industrialização de frutas, como a extração de polpas, sucos, extratos dentre outros produtos é importante forma de disponibilizar o acesso dos consumidores a essas frutas fora da época de colheita, ou em locais distantes das regiões produtoras, pois em geral, as frutas nativas apresentam um curto período de vida de prateleira, devido às características climatéricas dos frutos, com os mesmos entrando rapidamente em um processo de senescência, além de sua fragilidade, que torna difícil o manejo, o armazenamento e o transporte dos frutos, reduzindo em muito sua vida de prateleira pós-colheita. 0 processamento de frutas visando à extração da polpa geram resíduos, que são constituídos basicamente de sementes e cascas, e que podem possuir concentrações significativas de compostos bioativos, mas que na maioria das vezes acabam sendo descartados (OLIVEIRA et al., 2009). Esses compostos, por exemplo, podem ser extraídos e utilizados como substitutos dos antioxidantes sintéticos na indústria alimentícia.

Os antioxidantes são amplamente utilizados em alimentos para evitar a deterioração oxidativa de óleos e gorduras, a qual promove uma perda da qualidade nutricional e segurança dos alimentos. Desta forma, a adição de antioxidantes se faz necessária em alimentos para preservar suas qualidades sensoriais e nutricionais (FALOWO; FAYEMI; MUCHENJE, 2014; FRANKEL, 1996).

Estão disponíveis na literatura alguns estudos, avaliando a substituição dos antioxidantes sintéticos por antioxidantes extraídos de fontes naturais. Casagrande (2014), aplicou os compostos antioxidantes obtidos de coprodutos da uva oriundos da extração de vinho e suco, em linguiças de frango frescal, demonstrando eficácia na prevenção da oxidação lipídica. Brannan (2007), também obteve uma redução da oxidação em carne de coxas de frango moídas pelo uso de extrato de sementes de uva. Nissen et al. (2004), também observou a redução da oxidação lipídica em carne suína com o uso de antioxidantes extraídos de alecrim, chá verde, café e casca de uva.

Além da substituição de antioxidantes sintéticos por naturais, esses compostos podem ser utilizados visando benefícios a saúde também, quando utilizados para enriquecer o alimento com compostos bioativos, pois esses compostos podem ajudar o organismo a se proteger dos danos causados pelas 
espécies reativas ao oxigênio (HALLIWELL et al., 1995), também conhecidas como radicais livres.

Vários fatores devem ser levados em conta quando se extrai e se caracteriza os compostos bioativos de frutas. A forma e o tipo de solvente utilizado na extração, podem também levar a diferentes valores de quantificação. De acordo com Alothman; Bhat; Karim (2009) há diversos métodos estabelecidos para extração dos compostos fenólicos, sendo que em geral, tais protocolos de extração variam os solventes e as condições aplicadas, porém a natureza química do composto, o método utilizado e a técnica de caracterização são alguns fatores que também influenciam nos resultados obtidos. Pelo exposto, cada espécie de fruto apresenta características químicas únicas, sendo necessário um estudo focado no desenvolvimento de metodologia de extração que maximize a obtenção dos compostos de interesse daquele material.

Com base no exposto, o objetivo deste trabalho foi avaliar através de um planejamento fatorial, as variáveis tempo e temperatura para a extração de compostos fenólicos e avaliar a ação antioxidante do resíduo com potencial de coproduto da produção de polpa de araçá Ya-cy.

\section{MATERIAIS E MÉTODOS}

Os frutos de araçá Ya-Cy foram colhidos em pomar localizado na área experimental da Universidade Tecnológica Federal do Paraná, Câmpus Dois Vizinhos $\left(25^{\circ} 41^{\prime} 49,47^{\prime \prime} \mathrm{S} / 53^{\circ} 05^{\prime} 41,46^{\prime \prime} \mathrm{O}\right)$. Foram colhidos frutos com coloração amarela em ao menos metade da epiderme do fruto. Após a colheita, foram selecionados os frutos íntegros, sanitizados em solução de hipoclorito de sódio 200 ppm, e posteriormente enxaguados em água em temperatura ambiente. $\mathrm{Na}$ sequência os frutos foram processados em despolpadeira de frutas semi-industrial, com malha de $1 \mathrm{~mm}$ de diâmetro (DPT-75 - Tomasi, Brasil), com rendimento do subproduto (cascas e sementes) de $52,92 \%$.

Para realização do experimento, os subprodutos oriundos do processo de despolpa, constituído basicamente de cascas e sementes foi previamente congelado e posteriormente desidratado em liofilizador de bancada (LO2 - JJ científica, Brasil) por 36 horas. Após a completa secagem, as amostras foram moídas em moinho tipo Willye (TE-648 - Tecnal, Brasil) com peneira de 10 mesh. As amostras, previamente moídas, foram armazenadas em sacos de polietileno de baixa densidade $(\mathrm{PEBD})$ em freezer doméstico $\left(-18^{\circ} \mathrm{C}\right)$ por um período de 60 dias, até o momento da extração.

\section{PLANEJAMENTO FATORIAL PARA A EXTRAÇÃO DOS COMPOSTOS BIOATIVOS DO RESÍDUO DE ARAÇÁ}

Para o processo de extração de compostos bioativos fez-se uso de um planejamento fatorial $2^{2}$, com triplicata no ponto central, totalizando sete corridas experimentais. Sendo as variáveis independentes escolhidas: temperatura e tempo de extração. Os níveis reais e codificados da matriz do planejamento fatorial são apresentados na Tabela 1. 
O planejamento fatorial foi analisado utilizando o software Statistica 7.0 (Statsoft, USA). Para a análise dos efeitos fez-se uso do modelo linear apresentado na equação 1 :

$y=\beta_{0}+\beta_{1} x_{1}+\beta_{2} x_{2}+\beta_{12} x_{1} x_{2}+\varepsilon$

Onde: y é a variável dependente (resposta), $\beta$ é o coeficiente do modelo de regressão de cada termo, $x_{1}$ é a variável independente temperatura de extração $e$ $\mathrm{x}_{2}$ é a variável independente tempo de extração.

Tabela 1 - Variáveis do planejamento fatorial $2^{2}$

\begin{tabular}{ccccc}
\hline \multirow{2}{*}{ Corrida } & \multicolumn{2}{c}{ Níveis codificados } & \multicolumn{2}{c}{ Níveis decodificados } \\
\cline { 2 - 5 } & Temperatura ${ }^{\circ} \mathrm{C}$ & Tempo $(\mathrm{min})$ & Temperatura ${ }^{\circ} \mathrm{C}$ & Tempo $(\mathrm{min})$ \\
\hline 1 & -1 & -1 & 40 & 30 \\
2 & -1 & 1 & 40 & 90 \\
3 & 1 & -1 & 80 & 30 \\
4 & 1 & 1 & 80 & 90 \\
5 & 0 & 0 & 60 & 60 \\
6 & 0 & 0 & 60 & 60 \\
7 & 0 & 0 & 60 & 60 \\
\hline
\end{tabular}

\section{EXTRAÇÃO DE COMPOSTOS BIOATIVOS DE RESÍDUO DE ARAÇÁ}

Para o processo de extração, dois gramas de amostra liofilizada foram pesados em tubo de ensaio com tampa e adicionados de $25 \mathrm{~mL}$ do solvente de extração (água:acetona:etanol na proporção 1:1:1 v/v). Os tubos contendo o resíduo a ser extraído e o solvente, foram mantidos em banho-maria (SL150/14 - Solab, Brasil), conforme os tempos e temperaturas constantes na Tabela 1 , sendo agitados a cada cinco minutos. Após a extração, os extratos foram filtrados e armazenados a $4^{\circ} \mathrm{C}$ para posterior análise, conforme metodologia adaptada de Casagrande, (2014).

\section{DETERMINAÇÃO DE COMPOSTOS FENÓLICOS TOTAIS - CFT}

O teor de compostos fenólicos totais dos resíduos foi determinado pelo método espectrofotométrico de Folin-Ciocalteau, descrito por Singleton; Orthofer e Lamuela (1999), utilizando ácido gálico como padrão de referência para a realização da curva de calibração. $500 \mu \mathrm{L}$ de cada extrato em diluição adequada foi pipetado em tubos de ensaio, seguido da adição de $2,5 \mathrm{~mL}$ do reagente de FolinCiocalteau diluído (1:10 v/v), após 5 minutos, $2 \mathrm{~mL}$ de carbonato de sódio $(4 \% \mathrm{~m} / \mathrm{v}$ ) foram adicionados aos tubos, os mesmos foram incubados a temperatura ambiente e no escuro por 2 horas, procedendo-se posteriormente a leitura a 740

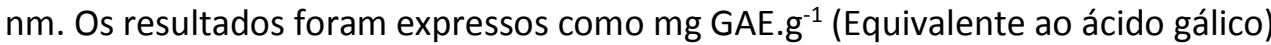
de amostra.

\section{DETERMINAÇÃO DE ATIVIDADE ANTIOXIDANTE PELO SEQUESTRO DE CÁTION $\mathrm{ABTS}^{+}$}

A atividade antioxidante pelo método $\mathrm{ABTS}^{\circ+}$ foi realizada conforme metodologia descrita por Re et al. (1999) e Rufino et al. (2007). A formação do radical $A B T S^{\bullet+}$ se procedeu pela reação da solução de $A B T S(7 \mathrm{mM})$ com persulfato 
de potássio $(140 \mathrm{mM})$, em temperatura ambiente e no escuro durante aproximadamente 14 horas. Para a realização da análise, $30 \mu \mathrm{L}$ do extrato em diluição adequada foram adicionados a tubos de ensaio, seguido da adição de 3,0 $\mathrm{mL}$ da solução de $\mathrm{ABTS}^{\circ+}$, com as leituras sendo realizadas a 734 nanômetros após 6 minutos de incubação. Os resultados foram expressos em $\mu$ mol TEAC. $\mathrm{g}^{-1}$, através da realização de uma curva de calibração com trolox.

\section{DETERMINAÇÃO DE ATIVIDADE ANTIOXIDANTE PELO MÉTODO DE REDUÇÃO DO FERRO - FRAP}

A atividade antioxidante pelo método da redução do ferro - FRAP (Ferric reducing Antioxidant Power) foi realizada de acordo com metodologia proposta por Rufino et al. (2006), o reagente FRAP foi preparado no momento da análise, através da combinação de $25 \mathrm{~mL}$ de tampão acetato $0,3 \mathrm{M}(\mathrm{pH} 3,6), 2,5 \mathrm{~mL}$ de TPTZ (,4,6-Tris(2-piridil)-s-triazina) dissolvido em solução de ácido clorídrico $40 \mathrm{mM}$ e $2,5 \mathrm{~mL}$ de solução de cloreto férrico $20 \mathrm{mM}$. Para a análise, $90 \mu \mathrm{L}$ de extrato previamente diluídos foram pipetados em tubos de ensaio, com posterior adição de $270 \mu \mathrm{L}$ de água destilada e $2,7 \mathrm{~mL}$ do reagente FRAP. Os tubos foram incubados em banho-maria a $37{ }^{\circ} \mathrm{C}$ por 30 minutos no escuro, com posterior leitura a 595

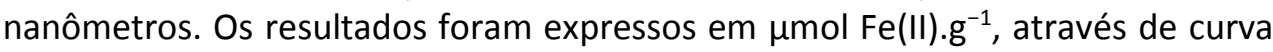
de calibração realizada com sulfato ferroso como padrão de referência.

\section{RESULTADOS E DISCUSSÃO}

O rendimento do processo de liofilização dos subprodutos da extração de polpa do araçá Ya-Cy foi de aproximadamente $20,40 \%$. A Tabela 2 apresenta as equações ajustadas para as variáveis dependentes analisadas.

Tabela 2- Equações dos modelos de regressão das análises de atividade antioxidante

\begin{tabular}{ccc} 
Resposta & Equação & $\mathrm{R}^{2}$ \\
\hline CFT & $y=12,48+0,60 x_{2}$ & 0,79 \\
DPPH $^{\circ}$ & $y=98,52-4,82 x_{1}+4,51 x_{1} x_{2}$ & 0,81 \\
ABTS $^{\circ+}$ & $y=175,08+9,70 x_{2}$ & 0,75 \\
FRAP & $y=375,69$ & - \\
\hline
\end{tabular}

NOTA: $y=$ Resposta; $x_{1}=$ Temperatura de extração; $x_{2}=$ Tempo de extração.

Para a resposta FRAP o modelo não apresentou bom ajuste, não sendo assim adequado na predição dos dados. Já para as variáveis dependentes CFT, DPPH• e $\mathrm{ABTS}^{\circ+}$, os modelos apresentaram um bom ajuste aos dados experimentais, com valores de coeficiente de determinação acima de $75 \%$, como pode ser observado também pela Tabela 3 que apresenta os valores observados e preditos pelo modelo para as variáveis dependentes $\mathrm{CFT}$, $\mathrm{DPPH}^{\bullet}$ e $\mathrm{ABTS}^{\circ+}$, sendo possível observar que o modelo ajustado se mostrou preditivo, devido às pequenas variações entre os valores observados e preditos pelo modelo matemático para as respostas de atividade antioxidante avaliadas.

Em relação à análise de FRAP, o valor médio observado foi de $375,69 \pm 16,80$ $\mu \mathrm{mol} \mathrm{Fe}(\mathrm{II}) \cdot \mathrm{g}^{-1}$. 
Para a variável CFT, os valores obtidos variaram entre 11,74 e $13,35 \mathrm{mg}$ de GAE. ${ }^{-1}$, sendo que o tempo de extração apresentou um efeito positivo, indicando que a melhor condição para a extração dos compostos fenólicos presentes nos subprodutos da produção de polpa do araçá Ya-Cy ocorre com o uso do maior tempo de extração avaliado, independentemente da temperatura utilizada, com como pode ser observado também pela Figura 1.

Tabela 3 - Resultados observados e preditos pelos modelos de regressão das análises de atividade antioxidante

\begin{tabular}{|c|c|c|c|c|c|c|}
\hline \multirow[t]{2}{*}{ Corrida } & \multicolumn{2}{|c|}{$\begin{array}{c}\text { Compostos Fenólicos } \\
\text { Totais } \\
\left(\mathrm{mg}^{\text {de }} \mathrm{GAE} \cdot \mathrm{g}^{-1}\right)\end{array}$} & \multicolumn{2}{|c|}{$\begin{array}{c}\mathrm{DPPH}^{\cdot} \\
\left(\mu \mathrm{mol} \text { trolox.g }{ }^{-1}\right)\end{array}$} & \multicolumn{2}{|c|}{$\begin{array}{c}\text { ABTS }^{\bullet+} \\
\left(\mu \mathrm{m} \text { TEAC. } \mathrm{g}^{-1}\right)\end{array}$} \\
\hline & Observado & Predito & Observado & Predito & Observado & Preditc \\
\hline 1 & 11,85 & 11,88 & 108,82 & 107,85 & 155,49 & 165,37 \\
\hline 2 & 11,74 & 11,88 & 90,16 & 89,18 & 170,25 & 165,37 \\
\hline 3 & 12,65 & 13,08 & 101,13 & 98,83 & 184,43 & 184,78 \\
\hline 4 & 13,35 & 13,08 & 100,50 & 98,20 & 180,13 & 184,78 \\
\hline 5 & 12,46 & 12,48 & 93,58 & 98,52 & 175,35 & 175,08 \\
\hline 6 & 12,51 & 12,48 & 96,34 & 98,52 & 179,06 & 175,08 \\
\hline 7 & 12,80 & 12,48 & 99,07 & 98,52 & 180,85 & 175,08 \\
\hline
\end{tabular}

Para a variável dependente $\mathrm{DPPH}^{\bullet}$, os resultados variaram de 90,16 a 108,82 $\mu \mathrm{mol}$ trolox. $\mathrm{g}^{-1}$, sendo observado pelo modelo que a temperatura de extração apresentou efeito significativo e negativo, e a interação linear tempo versus temperatura apresentando efeito significativo e positivo, demonstrando que a combinação dos menores níveis de tempo e temperatura (30 min, $40{ }^{\circ} \mathrm{C}$ ) favorecem a extração de compostos antioxidantes reativos ao radical orgânico DPPH $^{\circ}$, como pode ser observado na Figura 2.

Ao avaliarmos a resposta ABTS ${ }^{\circ+}$, os resultados variaram de 155,49 a 184,43 $\mu \mathrm{m}$ TEAC. $\mathrm{g}^{-1}$, com o tempo de extração apresentando efeito significativo e positivo, indicando conforme pode ser observado pela Figura 3, que os maiores tempos de extração promovem um aumento do potencial antioxidante do extrato pela captura do cátion $\mathrm{ABTS}^{\circ+}$, indiferentemente da temperatura estudada.

Biegelmeyer et al. (2011), obtiveram em frutos de araçá Ya-Cy valores de CFT de 2,93 mg de GAE.g ${ }^{-1}$ por grama de fruto fresco, sendo possível observar pelos valores obtidos no presente trabalho, quando transformados em base úmida $(2,39$ $-2,72 \mathrm{mg}$ de GAE. $\mathrm{g}^{-1}$ ) demonstram que os subprodutos do processo de extração da polpa possuem uma quantidade de compostos fenólicos remanescentes similar ao dos frutos inteiros, isso se deve ao resíduo ser constituído principalmente pela casca, que devido a sua coloração amarela possui considerável teor de compostos fenólicos, como antocianinas, responsáveis pela coloração da polpa e da epiderme dos frutos, conforme observado por Fetter et al. (2010).

Contreras-Calderón et al. (2011), obtiveram valores de FRAP e ABTS ${ }^{\bullet+}$ de 39,9

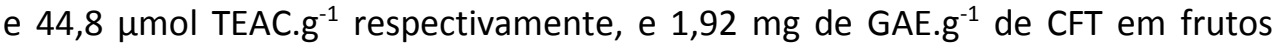
inteiros de araçá, valores menores que os observados por Biegelmeyer et al. (2011) e no presente estudo. Ainda segundo Biegelmeyer et al. (2011), essas variações entre autores podem ser atribuídas a maturidade e ao local de coleta dos frutos. 
Essas observações são corroboradas por Teixeira et al. (2016), ao estudarem a influência dos genótipos e do ano de colheita tanto do araçá vermelho quanto amarelo, sendo observadas diferenças tanto para os genótipos estudados quanto para os anos de colheita (entre os anos de 2008 a 2013) entre os parâmetros físicoquímicos dos frutos. Para o araçá amarelo, foram observados valores médios que variam entre 4 e $5 \mathrm{mg}$ de GAE. ${ }^{-1}$ de massa fresca de frutos, valores acima dos obtidos no presente trabalho, sendo inclusive observadas diferenças na capacidade antioxidante in vivo frente a cepas de $\mathrm{S}$. cereviseae entre os genótipos e anos de colheita, isso demonstra que há uma variabilidade dentro da própria espécie devido a fatores externos, como o clima ou mesmo o ponto de colheita dos frutos.

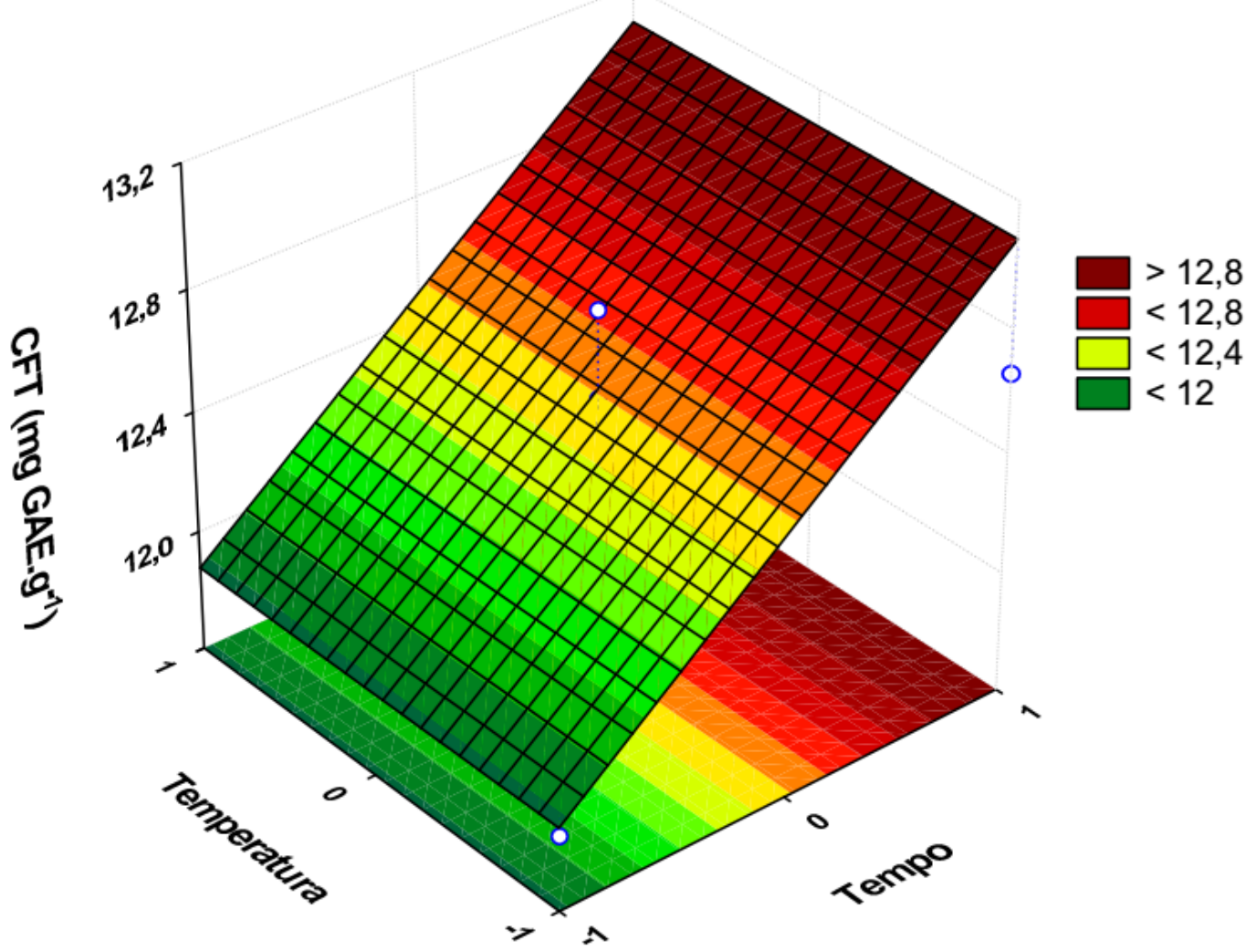

Figura 1- Superfície de resposta para a variável dependente Compostos Fenólicos Totais CFT em subprodutos da extração da polpa de araçá Ya-Cy.

Um fator que pode auxiliar a explicar os menores valores obtidos no presente trabalho, quando comparados a outros trabalhos da literatura pode ser justamente o ponto de colheita dos frutos, pois a colheita não se deu com o desenvolvimento total da cor amarela dos frutos, ponto que seria o considerado ideal para o consumo, mas sim quando os frutos apresentavam no mínimo $50 \%$ da epiderme com coloração amarela, como uma forma de reduzir possíveis danos provenientes da colheita e transporte, caso a colheita fosse feita mais próxima do ponto de desenvolvimento total da coloração amarela na epiderme dos frutos, esse fator poderia servir para aumentar ainda mais os teores de compostos bioativos encontrados nos resíduos. 


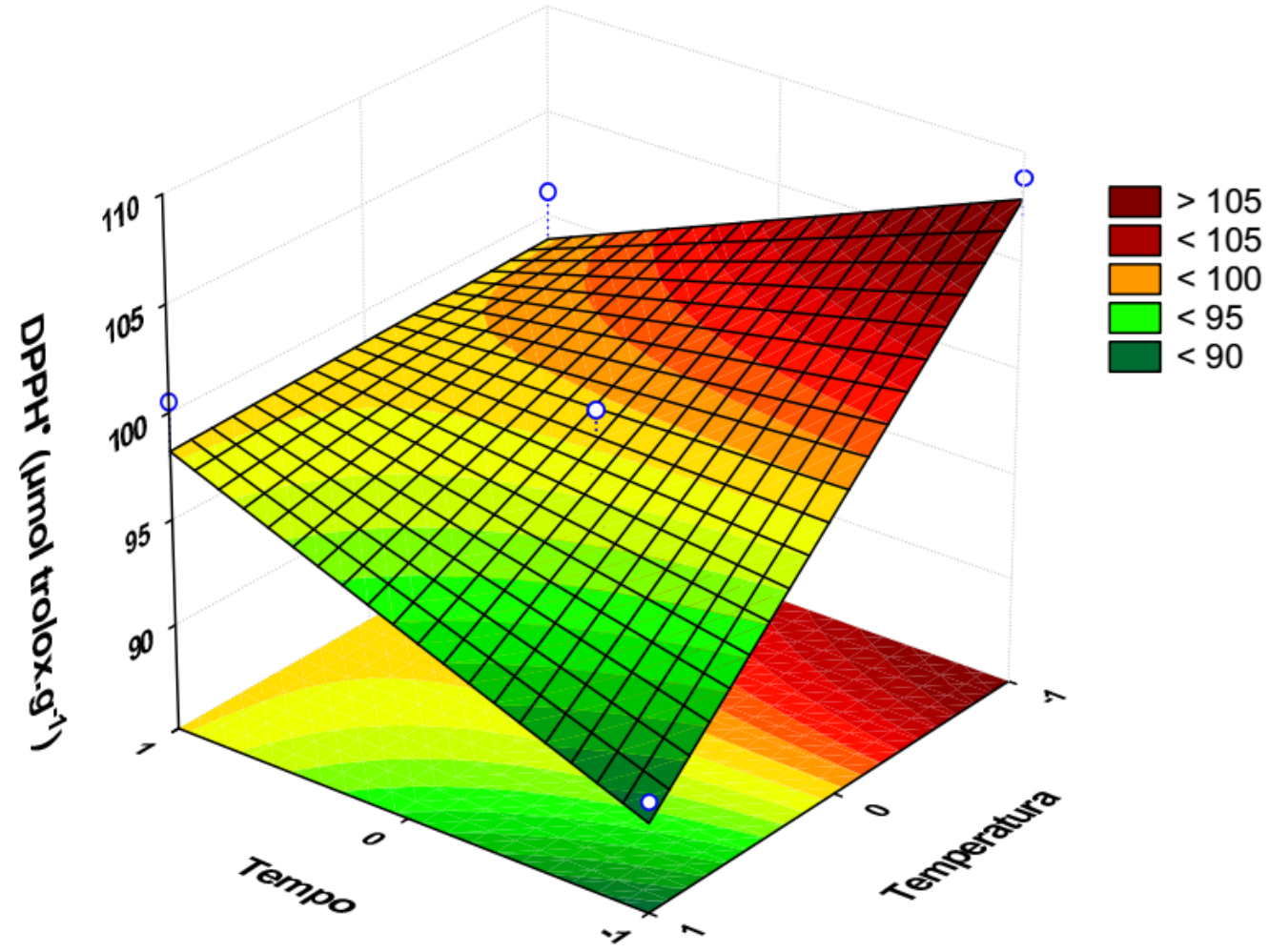

Figura 2- Superfície de resposta para a variável dependente $\mathrm{DPPH}^{\bullet}$ em subprodutos da extração da polpa de araçá Ya-Cy

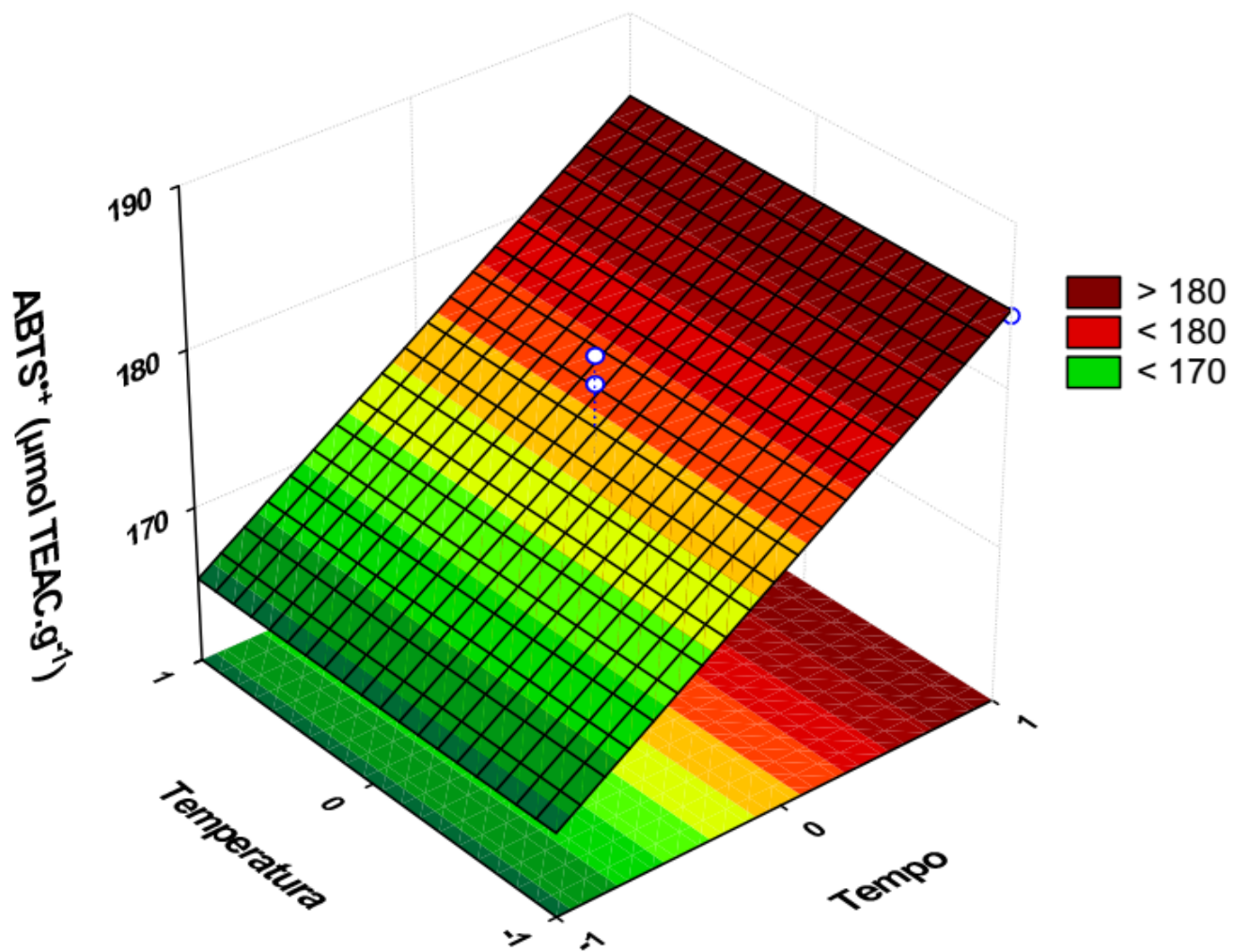

Figura 3 - Superfície de resposta para a variável dependente $\mathrm{ABTS}^{\bullet+}$ em derivados da extração da polpa de araçá Ya-Cy. 
Luximon-Ramma; Bahorun; Crozier (2003), ao estudarem os conteúdos de compostos fenólicos, vitamina $\mathrm{C}$ e atividade antioxidante de dezessete frutas exóticas comumente consumidas nas ilhas Maurício, dentre elas o araçá, os autores obtiveram valores de $\mathrm{CFT}, \mathrm{ABTS}^{\circ+}$ e FRAP para os frutos de araçá amarelo de 5,37 mg de GAE. $\mathrm{g}^{-1}, 45 \mu \mathrm{mol}$ TEAC. $\mathrm{g}^{-1}$ e $25 \mu \mathrm{mol}$ Fe(II) $\mathrm{g}^{-1}$ em massa de matéria fresca respectivamente, valores não muito diferentes aos observados no presente trabalho, quando convertidos em base úmida, em que foram obtidos valores de FRAP de 76,64 $\mu \mathrm{mol} \mathrm{Fe}(\mathrm{II}) \mathrm{g}^{-1}$, $\mathrm{DPPH}^{*}$ variando de 18,39 - 22,20 $\mu \mathrm{mol}$ trolox. $\mathrm{g}^{-1}$, e ABTS $^{*+}$ variando de $31,72-37,62 \mu \mathrm{mol}$ TEAC. $\mathrm{g}^{-1}$, demonstrando assim, que o subprodutos do processamento de frutos de araçá apresenta uma grande quantidade de compostos de interesse, com potencial antioxidante que estão presentes nas cascas e sementes do araçá. Salienta-se ainda que dentre as dezessete frutas estudadas, principalmente em compostos fenólicos e atividade antioxidante, ambos os frutos de araçá amarelo e vermelho apresentaram os maiores valores para essas análises, demonstrando o grande potencial de compostos bioativos presentes nesses frutos quando comparados a outras frutas comumente consumidas, como por exemplo, abacaxi, banana, manga dentre outras frutas que obtiveram valores até cerca de 10 vezes inferiores desses compostos de acordo com os autores.

Medina et al. (2011), ao estudar três acessos de araçazeiro amarelo obtiveram valores de CFT que variaram de 4,03 a 5,28 mg de GAE.g ${ }^{-1}$ e 5,32 a 7,72 mg de GAE. $\mathrm{g}^{-1}$ quando as amostras foram extraídas em água e acetona respectivamente, e valores de percentual de inibição do radical $\mathrm{DPPH}^{\bullet}$ de 15,98 a $27,63 \%$ e de 19,65 a $34,58 \%$ para a extração realizada com água e acetona respectivamente para esses acessos estudados. Observou-se ainda que esses extratos apresentaram atividade antioxidante in vivo em células se $S$. cereviseae e potencial antimicrobiano. Scur et al. (2016) ao avaliarem extratos aquosos e etanólicos de folhas de araçá, observaram maiores valores de captura do radical e menores valores de IC50 em extratos etanólicos quando comparados aos extratos aquosos, indicando que esses compostos são moderadamente polares, assim, sendo melhores extraídos em solventes com menor polaridade que a água, como no presente artigo, em que o solvente consiste de uma mistura ternária, resultando em diferentes graus de polaridade, podendo assim, promover uma melhor extração dos compostos de interesse.

Como demonstrando acima, há uma possível viabilidade e aplicabilidade da extração dos compostos bioativos dos resíduos da extração de polpa do araçá, bem como a importância do estudo da forma e dos solventes empregados no processo de extração para a maximização da extração.

\section{CONCLUSÕES}

O subproduto da produção de polpa de araçá Ya-Cy apresenta alto teor de compostos fenólicos e de atividade antioxidante, sendo um resíduo com potencial de ser um coproduto, pois é uma fonte potencial não explorada de compostos bioativos, com potencialidade para atuar como um substituto aos antioxidantes sintéticos, apresentando ainda a vantagem de ser um material pouco valorizado. 


\title{
Extraction of phenolic compounds and antioxidant activity in byproducts of araçá Ya-Cy (Psidium cattleianum Sabine) pulp production
}

\begin{abstract}
The araçá (Psidium cattleianum Sabine) is a native Brazilian fruit underexplored, however, some researches have been demonstrated that the araçá is a potential source of bioactive compounds, that was stimulated your consumption. Besides the in natura consumption, the araçá fruit can be processed, resulting in subproducts such as peels and seeds, that can contain a significant amount of bioactive compounds. The aim of this work was the extraction of this compounds. The araçá fruits were harvested, sanitized and processed in a pulp machine, the byproduct (peels and seeds) was freeze-dried and milled. The byproduct was extracted through a $2^{2}$ factorial design of experiment with triplicate in a central point, the evaluated factors were temperature and time of extraction: 40; 60 e 80 ${ }^{\circ} \mathrm{C}$ e 30; 60 e 90 minutes respectively. The used solvent was a ternary mixture of water:ethanol:acetone: $1: 1: 1(\mathrm{v} / \mathrm{v})$. The phenolics compounds and $\mathrm{ABTS}^{\circ+}$ content ranged from 11.74 to $13.35 \mathrm{mg} \mathrm{GAE} . \mathrm{g}^{-1}$ and 155,49 to $184,43 \mu \mathrm{mol}$ TEAC.g ${ }^{-1}$ respectively, the higher time level $(90 \mathrm{~min}$ ) improves the extraction, indifferently of temperature used. The values for $\mathrm{DPPH}^{*}$ analysis ranged from 90,16 to 108,82 $\mu \mathrm{mol}$ trolox. $\mathrm{g}^{-1}$, with the lower time and temperature values increasing the quantification $\left(30 \mathrm{~min}, 40{ }^{\circ} \mathrm{C}\right.$ ). The FRAP analysis don't present a good fit to the model, presenting the medium value of $375.69 \pm 16.80 \mu \mathrm{mol} \mathrm{Fe}(\mathrm{II}) \cdot \mathrm{g}^{-1}$. The byproduct of the araçá pulp extraction showed to be a potential source of bioactive compounds with antioxidant activity.
\end{abstract}

KEYWORDS: Factorial design; bioactive compounds; phenolic; araçá Ya-Cy. 


\section{REFERÊNCIAS}

ALOTHMAN, M.; BHAT, R.; KARIM, A. A. Antioxidant capacity and phenolic content of selected tropical fruits from Malaysia, extracted with different solvents. Food Chemistry, v. 115, n. 3, p. 785-788, 2009.

ANTUNES, L. E. C. Pequenas frutas: estratégias para o desenvolvimento, In: XIII Encontro nacional sobre fruticultura de clima temperado, Fraiburgo, Brasil: Anais, p. 115-122, 2013.

BIEGELMEYER, R.; ANDRADE, J. M. M.; ABOY, A. L.; APEL, M. A.; DRESCH, R. R, MARIN R.; RASEIRA, M. C. B.; HENRIQUES, A. M. Comparative analysis of the chemical composition and antioxidant activity of red (Psidium cattleianum) and yellow (Psidium cattleianum var. lucidum) strawberry guava fruit. Journal of Food Science, v. 76, n. 7, p. 991-996, 2011.

BRAND-WILLIAMS, W.; CUVELIER, M. E.; BERSET, C. Use of free radical method to evaluate antioxidant activity. LWT - Food Science and Technology, v. 28, n. 1, p. 25-30, 1995.

BRANNAN, R. G. Effect of grape seed extract on physicochemical properties of ground, salted, chicken thigh meat during refrigerated storage at different relative humidity levels. Journal of Food Science, v. 73, n. 1, p. 36-40, 2007.

CASAgRANDE, M. Avaliação do Potencial Antioxidante de Coprodutos de Indústrias de Suco de Uva e de Vinho Visando sua Aplicação em linguiça de Frango. 121 f. Dissertação (Mestrado em Tecnologia de Processos Químicos e Bioquímicos) - Universidade Tecnológica Federal do Paraná, Pato Branco, 2014.

CONTRERAS-CALDERÓN, J.; CALDERÓN-JAIMES, L.; GUERRA-HERNÁNDEZ, E.; GARCÍA-VILLANOVA, B. Antioxidant capacity, phenolic content and vitamin C in pulp, peel and seed from 24 exotic fruits from Colombia. Food Research International, v. 44, n. 7, p. 2047-2053, 2011.

FALOWO, A. B.; FAYEMI, P. O.; MUCHENJE, V. Natural antioxidants against lipidprotein oxidative deterioration in meat and meat products: A review. Food Research International, v. 64, p. 171-181, 2014.

FETTER, M. R.; VIZZOTTO, M.; CORBELINI, D. D.; GONZALEZ, T. N. Propriedades funcionais de araçá-amarelo, araçá-vermelho (Psidium cattleyanum Sabine) e araçá-pera ( $P$. acutangulum D. C.) cultivados em Pelotas/RS. Brazilian Journal of Food Technology. n. 15, p. 92-95, 2010. 
FRANKEL, E. N. Antioxidants in lipid foods and their impact on food quality. Food Chemistry, v. 57, n. 1, p. 51-55, 1996.

HALLIWELL, B.; AESCHBACH, R.; LÖLIGER, J.; ARUOMA, O. I. The characterization of antioxidants. Food and Chemical Toxicology, v. 33, n. 7, p. 601-617, 1995.

HAMINIUK, C. W. I.; SIERAKOWSKI, M. R.; VIDAL, J. R. M. B.; MASSON, M. L. Influence of temperature on the rheological behavior of whole araçá pulp (Psidium cattleianum Sabine). LWT - Food Science and Technology, v. 39, n. 4, p. 426-30, 2006.

LUXIMON-RAMMA, A.; BAHORUN, T.; CROZIER, A. Antioxidant actions and phenolic and vitamin $C$ contents of common Mauritian exotic fruits. Journal of the Science of Food and Agriculture, v. 83, n. 5, p. 496-502, 2003.

MATTOS, J. R. Myrtaceae do Rio Grande do Sul. Porto Alegre: CEUE, 1989. 721 p.

MEDINA, A. L; HAAS, L. I. R.; CHAVES, F. C.; SALVADOR, M.; ZAMBIAZI, R. C.; SILVA, W. P.; NORA, L.; ROMBALDI, C. V. Araçá (Psidium cattleianum Sabine) fruit extracts with antioxidant and antimicrobial activities and antiproliferative effect on human cancer cells. Food Chemistry, v. 128, n. 4, p. 916-922, 2011.

MENSOR, L. L.; MENEZES, F. S.; LEITÃO, G. G.; REIS, A. S.; SANTOS, T. C.; COUBE, C. S.; LEITÃO, S. G. Screening of brazilian plant extracts for antioxidant activity by the use of DPPH free radical method. Phytotheraphy Research, v. 15, n. 2, p. 127-130, 2001.

NISSEN, L. R.; BYRNE, D. V.; BERTELSEN, G.; SKIBSTED, L. H. The antioxidative activity of plant extracts in cooked pork patties as evaluated by descriptive sensory profiling and chemical analysis. Meat Science, v. 68, n. 3, p. 485-495, 2004.

OLIVEIRA, A. C.; VALENTIM, I. B.; GOULART, M. O. F.; SILVA, C. A.; BECHARA, E. J. H.; TREVISAN, M. T. S. Fontes vegetais naturais de antioxidantes. Química Nova, v. 32, n. 3, p. 689-702, 2009.

RASEIRA, M. C. B.; RASEIRA, A. Contribuição ao estudo do araçazeiro (Psidium cattleyanum). Pelotas: EMBRAPA/CPACT, 1996. $95 \mathrm{p}$.

RE, R.; PELLEGRINI, N.; PROTEGGENTE, A.; PANNALA, A.; YANG, M.; RICE-EVANS, assay. Free Radical Biology and Medicine, v. 26, n. 9-10, p.1231-1237, 1999. 
RUFINO, M. S. M.; ALVES, R. E.; BRITO, E. S.; MORAIS, S. M.; SAMPAIO, C. G.; PÉREZ-JIMÉNEZ, J.; SAURA-CALIXTO, F. D. Metodologia científica: Determinação da atividade antioxidante total em frutas pelo método de redução do ferro (FRAP). Comunicado Técnico 125. Embrapa - Fortaleza, CE, 2006. 4 p.

RUFINO, M. S. M.; ALVES, R. E.; BRITO, E. S.; MORAIS, S. M.; SAMPAIO, C. G.; PÉREZ-JIMÉNEZ, J.; SAURA-CALIXTO, F. D. Metodologia científica: Determinação da atividade antioxidante total em frutas pela captura do radical livre $\mathrm{ABTS}^{\circ+}$. Comunicado Técnico 128. Embrapa - Fortaleza, CE, 2007. 4 p.

SCUR, M. C.; PINTO, F. G. S.; PANDINI, J. A.; COSTA, W. F.; LEITE, C. W.; TEMPONI, L. G. Antimicrobial and antioxidante activity of essential oil and different plant extracts of Psidium cattleianum Sabine. Brazilian Journal of Biology, v. 76, n. 1, p. 101-108, 2016.

SINGLETON, V. L.; ORTHOFER, R.; LAMUELA-RAVENTÓS, R. M. Analysis of total phenols and other oxidation substrates and antioxidants by means of FolinCiocalteu reagent. Methods in Enzymology, v. 299, p. 152-178, 1999.

TEIXEIRA, A. M.; CHAVES, F. C.; FRANZON, R. C.; ROMBALDI, C. V. Influence of Genotype and Harvest Season on the Phytochemical Composition of Araçá (Psidium Cattleianum) Fruit. International Journal of Food and Nutritional Science, v. 3, n. 4, p. 1-7, 2016.

ZANELA, J. et al. Extração de compostos fenólicos e atividade antioxidante em derivados da produção de polpa de araçá Ya-Cy (Psidium cattleianum Sabine). Brazilian Journal of Food Research, Campo Mourão, v. 9, n. 4, p. 14-26, out./dez. 2018. Disponível em: https://periodicos.utfpr.edu.br/rebrapa

Correspondência:

Juliano Zanela

Universidade Tecnológica Federal do Paraná, Câmpus Dois Vizinhos, Estrada para Boa Esperança, Km 04 CEP: 85660-000, Dois Vizinhos, Paraná, Brasil.

Direito autoral: Este artigo está licenciado sob os termos da Licença Creative Commons-Atribuição 4.0 Internacional. 